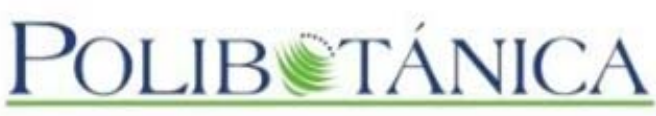

\title{
Polibotánica
}

ISSN electrónico: 2395-9525

polibotanica@gmail.com

Instituto Politécnico Nacional

México

http://www.polibotanica.mx

\section{HELECHOS Y LICOFITAS: ACTUALIZACIÓN DE LA FLORA DEL VALLE DE LERMA - SALTA, ARGENTINA.}

\section{FERNS AND LYCOPHYTES: AN UPDATE ON THE FLORA OF THE VALLE DE LERMA-SALTA, ARGENTINA.}

Jarsun, A.M., J.C. Chambi, D.G. Jaimez, D.A. Cacharani y O.G. Martínez.

HELECHOS Y LICOFITAS: ACTUALIZACIÓN DE LA FLORA DEL VALLE DE LERMA SALTA, ARGENTINA.

FERNS AND LYCOPHYTES: AN UPDATE ON THE FLORA OF THE VALLE DE LERMA- SALTA, ARGENTINA.

\section{POLIBETANICA}

Instituto Politécnico Nacional
Núm. 49: 1-14 México. Enero 2020

DOI: 10.18387 polibotanica.49.1

Este es un artículo de acceso abierto bajo la licencia Creative Commons 4.0 Atribución-No Comercial (CC BY-NC 4.0 Internacional). 


\section{HELECHOS Y LICOFITAS: ACTUALIZACIÓN DE LA FLORA DEL VALLE DE LERMA - SALTA, ARGENTINA.}

\section{FERNS AND LYCOPHYTES: AN UPDATE ON THE FLORA OF THE VALLE DE LERMA-SALTA, ARGENTINA.}

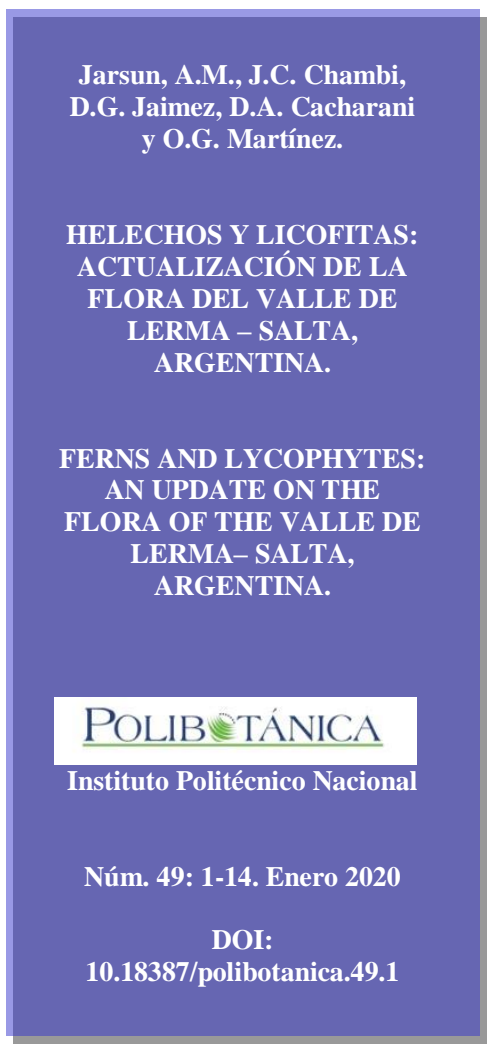

\author{
A.M. Jarsun \\ Instituto de Bio y Geociencias del Noroeste Argentino (IBIGEO-CONICET), \\ Universidad Nacional de Salta, Av. Bolivia 5150, 4400 Salta, Argentina \\ J.C. Chambi \\ Herbario MCNS, Facultad de Ciencias Naturales, \\ Universidad Nacional de Salta, Av. Bolivia 5150, 4400 Salta, Argentina. \\ D.G. Jaimez \\ D.A. Cacharani \\ Instituto de Bio y Geociencias del Noroeste Argentino (IBIGEO-CONICET), \\ Universidad Nacional de Salta, Av. Bolivia 5150, 4400 Salta, Argentina. \\ O.G. Martínez / martinezog@gmail.com \\ Instituto de Bio y Geociencias del Noroeste Argentino (IBIGEO-CONICET); \\ Herbario MCNS, Facultad de Ciencias Naturales, \\ Universidad Nacional de Salta, Av. Bolivia 5150, 4400 Salta, Argentina.
}

RESUMEN: El Valle de Lerma se encuentra en el centro de la provincia de Salta, Argentina. Su vegetación corresponde a dos dominios fitogeográficos, amazónico y chaqueño. Se presenta un listado actualizado de la diversidad de helechos y licófitas como resultado de nuevas colectas y revisión de ejemplares de herbarios y bibliografía específica. Se registraron 115 taxones, 107 helechos y 8 licófitas, pertenecientes a 16 y 2 familias respectivamente, lo que corresponde a un incremento de 22 nuevos taxones respecto a la flora original. Tres registros se citan por primera vez para la región noroeste de Argentina, Microgramma vaccinifolia, Pellaea atropurpurea y Pteris multifida. Las tres familias mejor representadas son, Pteridaceae con 34 taxones, Polypodiaceae con 19, y Aspleniaceae con 14. De los 115 taxones identificados, 110 pertenecen a la flora nativa y cinco son especies naturalizadas.

Palabras clave: Argentina, Dominio Amazónico, Dominio Chaqueño, Polypodiaceae, Pteridaceae.

ABSTRACT: The Lerma Valley is located in the center of the province of Salta, Argentina. Its vegetation corresponds to two phytogeographic domains, Amazonian domain and Chaco domain. An updated list of the diversity of ferns and lycophytes is presented as a result of new collections and a review of herbarium specimens and specific bibliography. We registered 115 taxa, 107 ferns, and 8 lycophytes, belonging to 16 and 2 families respectively, which correspond to an increase of 22 new taxa compared to the original flora. Three records are cited for the first time for the northwest region of Argentina, Microgramma vaccinifolia, Pellaea atropurpurea and Pteris multifida. The three best-represented families are Pteridaceae with 34 taxa, Polypodiaceae with 19, and Aspleniaceae with 14. Of the 115 taxa identified, 110 belong to the native flora and five are naturalized species.

Key words: Argentina, Amazonian domain, Chaco domain, Polypodiaceae, Pteridaceae. 


\section{INTRODUCCIÓN}

La provincia de Salta se encuentra en el noroeste de Argentina, limita al norte con Bolivia y al oeste con Chile. El Valle de Lerma se ubica en la parte media de la provincia de Salta, ocupa una franja de unos $130 \mathrm{~km}$ de longitud y $63 \mathrm{~km}$ de ancho, con una superficie de ca. $4500 \mathrm{~km}^{2}$ (Fig. 1). Este valle intermontano de origen Cuaternario forma parte del sistema de las Sierras Subandinas; el piso del valle tiene una elevación promedio de $1200 \mathrm{~m}$ snm, mientras que los cordones montañosos del este se extienden desde los 1600 hasta los $2800 \mathrm{~m}$ snm, y los del oeste superan los $4500 \mathrm{~m}$ snm (Baudino, 1996). En la parte más baja del valle se ubica la ciudad de Salta que alberga más de 600.000 habitantes.

La vegetación del Valle de Lerma corresponde a los dominios fitogeográficos chaqueño y amazónico según Cabrera \& Willink (1973), o chaco seco y yungas australes según Olson, Dinerstein, Wikramanayake, Burgess, Powell, et al. (2001). Las unidades de vegetación fueron descritas por Martínez \& Prado (2013), quienes destacan cinco unidades en orden creciente de altitud: bosque chaqueño serrano, selva pedemontana, selva montana, bosque montano y pastizal de altura; correspondiendo la selva pedemontana a los bosques secos estacionales neotropicales (Prado \& Gibbs, 1993).

La diversidad de helechos y licófitas del Valle de Lerma fue abordada por de la Sota \& Martínez (1998, 2000), de la Sota, Ponce, Martínez, Giudice \& Michelena (2001), Ganem, Giudice, Martínez \& de la Sota (2007), Martínez (1995, 1996a, 1996b, 1997, 1998), Martínez \& Cacharani (2011), Martínez \& de la Sota (2000a, 2000b, 2005), Martínez, de la Sota \& Aquino (2006), Martínez, de la Sota \& Narváez (2003, 2004), Ponce \& Martínez (2008, 2012), quienes citan siete especies de licófitas y 88 taxones específicos e infraespecíficos de helechos, excepto la familia Blechnaceae. Recientes exploraciones en la zona y avances en la taxonomía, obligan a actualizar el conocimiento de la diversidad regional de estas plantas.

\section{MATERIAL Y MÉTODOS}

El material estudiado proviene del Valle de Lerma (Salta, Argentina) (fig. 1), corresponde a colectas realizadas por los autores y a ejemplares depositados en los herbarios BAB, CORD, LIL, LP, MCNS y SI (Thiers, en constante actualización).

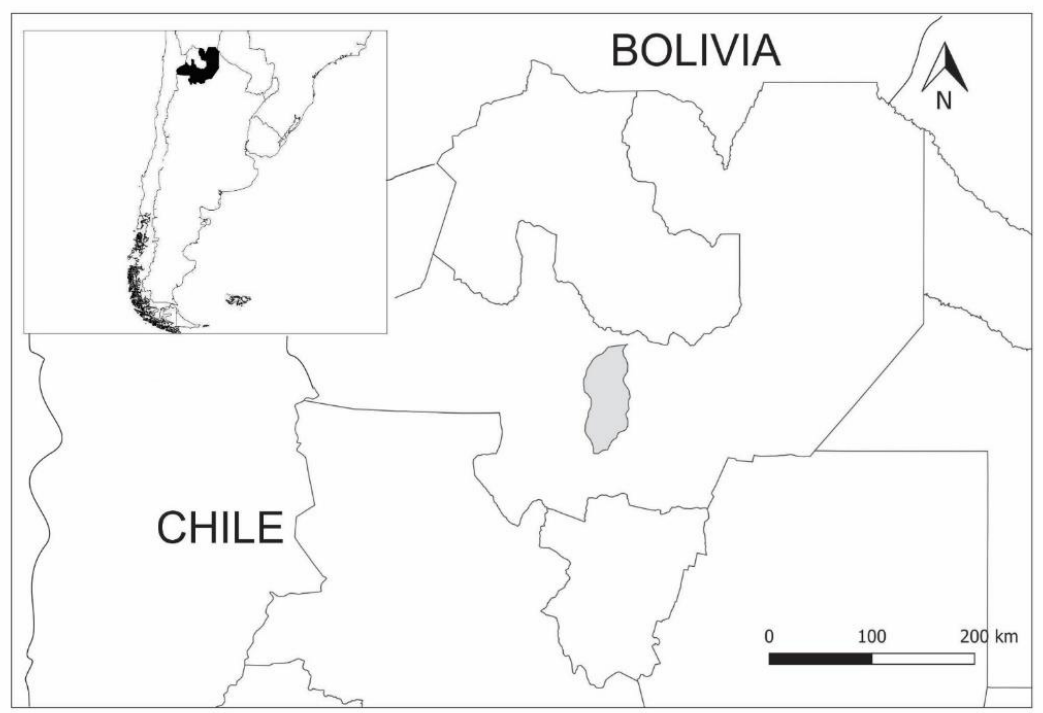

Fig. 1. Ubicación del Valle de Lerma en la provincia de Salta, Argentina. 
La identificación taxonómica se realizó consultando los tipos nomenclaturales a través de las imágenes digitales disponibles en Jstor Plant Science (http://plants.jstor.org), y mediante bibliografía específica para cada grupo taxonómico.

Para cada taxón se indica la familia a la que pertenece, el dominio fitogeográfico que habita la planta, el ejemplar de referencia señalando el herbario donde se encuentra depositado, y se indica con asterisco (*) a los nuevos registros. El mapa se confeccionó mediante Q-Gis ver 2.18.9.

\section{REsultados}

La diversidad de helechos y licófitas existentes en el Valle de Lerma se presentan en la tabla 1 y figura 2.

Tabla 1. Diversidad de helechos y licófitas del Valle de Lerma, señalando el dominio fitogeográfico que habita cada taxón (DA: dominio amazónico, DA-DCH: dominio amazónico y dominio chaqueño, DCH: dominio chaqueño)

(* nuevos registros respecto de la flora original).

\begin{tabular}{|c|c|c|c|}
\hline \multicolumn{4}{|c|}{ LICÓFITAS } \\
\hline Familia & Nombre actual & Dominio & Ejemplar de Referencia \\
\hline Lycopodiaceae & Austrolycopodium erectum (Phil.) Holub* & DA & Aquino 364 (MCNS) \\
\hline Lycopodiaceae & Phlegmariurus saururus (Lam.) B. Øllg. & DA-DCH & Sleumer \& Vervoorst 2910 (MCNS) \\
\hline Lycopodiaceae & Phlegmariurus mandiocanus (Raddi) B. Øllg. & DA & Venturi 9822 (LIL) \\
\hline Lycopodiaceae & Lycopodium clavatum L. & DA & Palací 998 (MCNS) \\
\hline Selaginellaceae & Selaginella microphylla (Kunth) Spring & DA-DCH & Martínez 278 (MCNS) \\
\hline Selaginellaceae & Selaginella novae-hollandiae (Sw.) Spring & DA & Novara 3192 (MCNS) \\
\hline Selaginellaceae & Selaginella peruviana (Milde) Hieron. & DA-DCH & Martínez 314 (MCNS) \\
\hline Selaginellaceae & Selaginella sellowii Hieron. & DA-DCH & Martínez \& Prado 3308 (MCNS) \\
\hline
\end{tabular}

\section{HELECHOS}

\begin{tabular}{llll}
\hline \multicolumn{1}{c}{ Familia } & \multicolumn{1}{c}{ Nombre actual } & Dominio & Ejemplar de Referencia \\
\hline Anemiaceae & $\begin{array}{l}\text { Anemia tomentosa (Savigny) Sw. var. } \\
\text { anthriscifolia } \text { (Schrad.) Mickel } \\
\text { Anemia australis (Mickel) M. Kessler \& } \\
\text { A.R. Sm. }\end{array}$ & DA-DCH & Narváez 66 (MCNS) \\
Aspleniaceae & Asplenium argentinum Hieron. & DA & Novara 7894 (MCNS) \\
Aspleniaceae & Asplenium achalense Hieron. & DA & Aquino 209 (MCNS) \\
\hline
\end{tabular}




\section{HELECHOS}

\begin{tabular}{|c|c|c|c|}
\hline Familia & Nombre actual & Dominio & Ejemplar de Referencia \\
\hline Aspleniaceae & Asplenium auritum $\mathrm{Sw}$. & DA & Martínez 232 (MCNS) \\
\hline Aspleniaceae & Asplenium cuspidatum Lam* & DA & Venturi 8290 (SI) \\
\hline Aspleniaceae & Asplenium depauperatum Fée & DA-DCH & Figueroa 132 (MCNS) \\
\hline Aspleniaceae & Asplenium gilliesii Hook. & DA-DCH & Martínez 581 (MCNS) \\
\hline Aspleniaceae & Asplenium harpeodes Kunze* & DA & Novara 8090 (CORD) \\
\hline Aspleniaceae & Asplenium lilloanum de la Sota & DA & Aquino 207 (MCNS) \\
\hline Aspleniaceae & Asplenium lorentzii Hieron. & DA & $\begin{array}{l}\text { Abbiatti \& Claps } 116 \\
\text { (MCNS) }\end{array}$ \\
\hline Aspleniaceae & Asplenium monanthes L. & DA & Novara 3537 (MCNS) \\
\hline Aspleniaceae & Asplenium praemorsum $\mathrm{Sw}$. & DA & Martínez 1144 (MCNS) \\
\hline Aspleniaceae & Asplenium pumilum $\mathrm{Sw}$. & DA & Palací 918 (MCNS) \\
\hline Aspleniaceae & Asplenium resiliens Kunze & DA & Martínez 291 (MCNS) \\
\hline Aspleniaceae & Asplenium squamosum $\mathrm{L}$. & DA & $\begin{array}{l}\text { Martínez \& Aquino } 1058 \\
\text { (MCNS) }\end{array}$ \\
\hline Athyriaceae & $\begin{array}{l}\text { Diplazium lilloi (Hicken) R.M. Tryon \& } \\
\text { A.F. Tryon }\end{array}$ & DA & Martínez 872 (MCNS) \\
\hline Blechnaceae & $\begin{array}{l}\text { Austroblechnum penna-marina (Poir.) } \\
\text { Gasper \& V.A.O. Dittrich* }\end{array}$ & DA & Novara 10132 (MCNS) \\
\hline Blechnaceae & Blechnum austrobrasilianum de la Sota* & DA & Chambi 192 (MCNS) \\
\hline Blechnaceae & $\begin{array}{l}\text { Blechnum occidentale L. var. } \\
\text { occidentale* }^{*}\end{array}$ & DA & $\begin{array}{l}\text { Martínez \& Prado } 1880 \\
\text { (MCNS) }\end{array}$ \\
\hline Blechnaceae & $\begin{array}{l}\text { Cranfillia caudata (Baker) V.A.O. } \\
\text { Dittrich \& Gasper* }\end{array}$ & DA & Palací 765 (MCNS) \\
\hline Cystopteridaceae & Cystopteris diaphana (Bory) Blasdell & DA & Martínez 1365 (MCNS) \\
\hline Dennstaedtiaceae & $\begin{array}{l}\text { Pteridium esculentum (G. Forst.) } \\
\text { Cockayne var. arachnoideum }\end{array}$ & DA & $\begin{array}{l}\text { Cabrera \& Schawabe } 223 \\
\text { (LP) }\end{array}$ \\
\hline Dennstaedtiaceae & $\begin{array}{l}\text { Dennstaedtia glauca (Cav.) C. Chr. ex } \\
\text { Looser }\end{array}$ & DA & $\begin{array}{l}\text { Martínez \& Novara } 562 \\
\text { (MCNS) }\end{array}$ \\
\hline Dennstaedtiaceae & Dennstaedtia globulifera (Poir.) Hieron. & DA & Martínez 139 (MCNS) \\
\hline Dennstaedtiaceae & Hypolepis poeppigii (Kunze) R. Rodr. & DA & Guerra R. 102 (MCNS) \\
\hline Dryopteridaceae & $\begin{array}{l}\text { Ctenitis submarginalis (Langsd. \& } \\
\text { Fisch.) Ching var. submarginalis }\end{array}$ & DA & Martínez 1774 (MCNS) \\
\hline Dryopteridaceae & Dryopteris patula (Sw.) Underw. & DA & Martínez 1928 (MCNS) \\
\hline Dryopteridaceae & Dryopteris wallichiana (Spreng.) Hyl. & DA & Narváez et al. 127 (MCNS) \\
\hline
\end{tabular}




\section{HELECHOS}

\begin{tabular}{|c|c|c|c|}
\hline Familia & Nombre actual & Dominio & Ejemplar de Referencia \\
\hline Dryopteridaceae & Elaphoglossum crassipes (Hieron.) Diels & $\mathrm{DA}$ & Aquino 366 (MCNS) \\
\hline Dryopteridaceae & Elaphoglossum gayanum (Fée) T. Moore & DA & Aquino 319 (MCNS) \\
\hline Dryopteridaceae & $\begin{array}{l}\text { Elaphoglossum spathulatum (Bory) } \mathrm{T} \text {. } \\
\text { Moore }\end{array}$ & DA & Aquino 317 (MCNS) \\
\hline Dryopteridaceae & Elaphoglossum yungense de la Sota & DA & $\begin{array}{l}\text { Palací \& Mosqueira } 1116 \\
\text { (MCNS) }\end{array}$ \\
\hline Dryopteridaceae & $\begin{array}{l}\text { Megalastrum fugaceum R.C. Moran, J. } \\
\text { Prado \& Sundue* }\end{array}$ & DA & Martínez 149 (MCNS) \\
\hline Dryopteridaceae & $\begin{array}{l}\text { Polystichum montevidense (Spreng.) } \\
\text { Rosenst. }\end{array}$ & DA & Narváez 77 (MCNS) \\
\hline Dryopteridaceae & $\begin{array}{l}\text { Polystichum platyphyllum (Willd.) C. } \\
\text { Presl var. platyphyllum }\end{array}$ & DA & Palací 875 (MCNS) \\
\hline Dryopteridaceae & $\begin{array}{l}\text { Polystichum pycnolepis (Kunze ex } \\
\text { Klozstch) Hieron* }\end{array}$ & DA-DCH & Martínez 165 (MCNS) \\
\hline Equisetaceae & Equisetum bogotense Kunth & DA-DCH & Pastore 2361 (LP) \\
\hline Equisetaceae & Equisetum giganteum $\mathrm{L}$. & DA-DCH & Martínez 122 (MCNS) \\
\hline Hymenophyllaceae & $\begin{array}{l}\text { Hymenophyllum cordobense (Hieron.) } \\
\text { C. Larsen \& Arana }\end{array}$ & DA & Palací 149 (MCNS) \\
\hline Ophioglossaceae & $\begin{array}{l}\text { Ophioglossum crotalophoroides Walter } \\
\text { subsp. crotalophoroides }\end{array}$ & DA & $\begin{array}{l}\text { Sleumer \& Vervoorst } 2897 \\
\text { (LIL) }\end{array}$ \\
\hline Ophioglossaceae & Ophioglossum nudicaule L. f. & DA & $\begin{array}{l}\text { Martínez \& Novara } 540 \\
\text { (MCNS) }\end{array}$ \\
\hline Ophioglossaceae & Ophioglossum reticulatum $\mathrm{L}$. & DA & Nuñez 498 (MCNS) \\
\hline Ophioglossaceae & Sceptridium schaffneri (Underw.) Lyon & DA & $\begin{array}{l}\text { Sleumer \& Vervoorst } 2896 \\
\text { (LIL) }\end{array}$ \\
\hline Polypodiaceae & $\begin{array}{l}\text { Campyloneurum aglaolepis (Alston) de } \\
\text { la Sota }\end{array}$ & DA & $\begin{array}{l}\text { Legname \& Cuezzo 6057C } \\
\text { (LP) }\end{array}$ \\
\hline Polypodiaceae & $\begin{array}{l}\text { Campyloneurum angustifolium (Sw.) } \\
\text { Fée* }\end{array}$ & DA & Chambi et al. 242 (MCNS) \\
\hline Polypodiaceae & $\begin{array}{l}\text { Campyloneurum angustipaleatum } \\
\text { (Alston) M. Mey. ex Lellinger* }\end{array}$ & DA & Martínez 1604 (MCNS, SI) \\
\hline Polypodiaceae & $\begin{array}{l}\text { Campyloneurum lorentzii (Hieron.) } \\
\text { Ching }\end{array}$ & DA & $\begin{array}{l}\text { Martínez \& Prado } 3320 \\
\text { (MCNS) }\end{array}$ \\
\hline Polypodiaceae & $\begin{array}{l}\text { Melpomene peruviana (Desv.) A.R. Sm. } \\
\text { \& R.C. Moran }\end{array}$ & DA & $\begin{array}{l}\text { Martínez \& Prado } 1906 \\
\text { (MCNS) }\end{array}$ \\
\hline Polypodiaceae & Microgramma mortoniana de la Sota* & DA & $\begin{array}{l}\text { Martínez et al. } 3348 \\
\text { (MCNS) }\end{array}$ \\
\hline Polypodiaceae & $\begin{array}{l}\text { Microgramma squamulosa (Kaulf.) de la } \\
\text { Sota }\end{array}$ & DA & $\begin{array}{l}\text { Martínez \& Prado } 3327 \\
\text { (MCNS) }\end{array}$ \\
\hline
\end{tabular}




\section{HELECHOS}

\begin{tabular}{|c|c|c|c|}
\hline Familia & Nombre actual & Dominio & Ejemplar de Referencia \\
\hline Polypodiaceae & $\begin{array}{l}\text { Microgramma vacciniifolia (Langsd. \& } \\
\text { Fisch.) Copel.* }\end{array}$ & $\overline{\mathrm{DA}}$ & Chambi 776 (MCNS) \\
\hline Polypodiaceae & Pecluma oranense (de la Sota) de la Sota & DA & Palací 1107 (MCNS) \\
\hline Polypodiaceae & Pecluma venturii (de la Sota) M.G. Price & DA & Palací 166 (LP) \\
\hline Polypodiaceae & $\begin{array}{l}\text { Phlebodium areolatum (Humb. \& } \\
\text { Bonpl. Ex Willd.) J. Sm. }\end{array}$ & DA & $\begin{array}{l}\text { Núñez \& Marmol } 450 \\
\text { (MCNS) }\end{array}$ \\
\hline Polypodiaceae & Pleopeltis bryopoda (Maxon) de la Sota & DA & $\begin{array}{l}\text { Martínez \& Novara } 565 \\
\text { (MCNS) }\end{array}$ \\
\hline Polypodiaceae & $\begin{array}{l}\text { Pleopeltis macrocarpa (Bory ex Willd.) } \\
\text { Kaulf. }\end{array}$ & DA & Martínez 555 (MCNS) \\
\hline Polypodiaceae & $\begin{array}{l}\text { Pleopeltis minima (Bory) J. Prado \& } \\
\text { R.Y. Hirai }\end{array}$ & DA & $\begin{array}{l}\text { Martínez \& Prado } 2149 \\
\text { (MCNS) }\end{array}$ \\
\hline Polypodiaceae & $\begin{array}{l}\text { Pleopeltis pinnatifida Gillies ex Hook. } \\
\text { \& Grev. }\end{array}$ & DA & Martínez 427 (MCNS) \\
\hline Polypodiaceae & Pleopeltis tweediana (Hook.) A.R. Sm & DA & $\begin{array}{l}\text { Martínez \& Prado } 2142 \\
\text { (MCNS) }\end{array}$ \\
\hline Polypodiaceae & $\begin{array}{l}\text { Serpocaulon australe D. Sanín, J.C. } \\
\text { Ospina, I.O. Moura \& Salino* }\end{array}$ & DA & Jarsun et al. 205 (MCNS) \\
\hline Polypodiaceae & Serpocaulon gilliesii (C. Chr.) A.R. Sm. & DA & Novara 9394 (MCNS) \\
\hline Polypodiaceae & Serpocaulon triseriale (Sw.) A.R. Sm.* & DA & Ramos s.n. (MCNS 12747) \\
\hline Pteridaceae & Adiantopsis chlorophylla (Sw.) Fée & DA-DCH & Palací 170 (MCNS) \\
\hline Pteridaceae & $\begin{array}{l}\text { Adiantopsis orbignyana }(\mathrm{Kuhn}) \text { Ponce \& } \\
\text { Scataglini* }\end{array}$ & DA & Novara 4979 (MCNS) \\
\hline Pteridaceae & Adiantum digitatum Hook. & DA & $\begin{array}{l}\text { Sleumer \& Vervoorst } 2764 \\
\text { (BAB) }\end{array}$ \\
\hline Pteridaceae & Adiantum lorentzii Hieron. & DA & $\begin{array}{l}\text { Martínez \& Chambi } 1972 \\
\text { (MCNS) }\end{array}$ \\
\hline Pteridaceae & Adiantum orbignyanum Mett. ex Kuhn & DA & Martínez 539 (MCNS) \\
\hline Pteridaceae & Adiantum peruvianum Klotzch.* & DA & Ramos s.n. (MCNS 12746) \\
\hline Pteridaceae & Adiantum raddianum C. Presl. & DA & $\begin{array}{l}\text { Novara \& Neumann } 3195 \\
\text { (MCNS) }\end{array}$ \\
\hline Pteridaceae & Adiantum poiretii Wikstr. & DA & $\begin{array}{l}\text { Martínez \& Prado } 3323 \\
\text { (MCNS) }\end{array}$ \\
\hline Pteridaceae & Anogramma lorentzii (Hieron.) Diels* & DA & Mezza Torres 797 (Ctes) \\
\hline
\end{tabular}




\section{HELECHOS}

\begin{tabular}{|c|c|c|c|}
\hline Familia & Nombre actual & Dominio & Ejemplar de Referencia \\
\hline Pteridaceae & Argyrochosma nivea (Poir.) Windham & $\mathrm{DCH}$ & Martínez 320 (MCNS) \\
\hline Pteridaceae & $\begin{array}{l}\text { Argyrochosma tenera (Gillies ex Hook.) } \\
\text { M. Kessler \& A.R. Sm. }\end{array}$ & $\mathrm{DCH}$ & Martínez 313 (MCNS) \\
\hline Pteridaceae & $\begin{array}{l}\text { Cheilanthes buchtienii (Rosenst.) R.M. } \\
\text { Tryon }\end{array}$ & DA-DCH & Martínez 166 (MCNS) \\
\hline Pteridaceae & Cheilanthes pilosa Goldm. & DA-DCH & Palací 912 (MCNS) \\
\hline Pteridaceae & $\begin{array}{l}\text { Doryopteris concolor (Langsd. \& Fisch.) } \\
\text { Kuhn }\end{array}$ & DA-DCH & Martínez 155 (MCNS) \\
\hline Pteridaceae & Doryopteris lorentzii (Hieron.) Diels & DA-DCH & $\begin{array}{l}\text { Martínez \& Prado } 3330 \\
\text { (MCNS) }\end{array}$ \\
\hline Pteridaceae & $\begin{array}{l}\text { Myriopteris aurea (Poir.) Grusz \& } \\
\text { Windham }\end{array}$ & DA-DCH & Novara 1910 (MCNS) \\
\hline Pteridaceae & $\begin{array}{l}\text { Myriopteris microphylla (Sw.) Grusz \& } \\
\text { Windham }\end{array}$ & DA & Palací 431 (MCNS) \\
\hline Pteridaceae & $\begin{array}{l}\text { Pityrogramma calomelanos (L.) Link } \\
\text { var. austroamericana (Domin) Farw. }\end{array}$ & DA-DCH & Novara 1919 (MCNS) \\
\hline Pteridaceae & Pityrogramma trifoliata (L.) R.M. Tryon & DA-DCH & Martínez 124 (MCNS) \\
\hline Pteridaceae & Polytaenium lineatum (Sw.) Sm. & DA & Martínez 1393 (MCNS) \\
\hline Pteridaceae & Pteris cretica $\mathrm{L}$. & DA & $\begin{array}{l}\text { Martínez \& Chambi } 1964 \\
\text { (MCNS) }\end{array}$ \\
\hline Pteridaceae & Pteris deflexa Link & DA & Martínez 126 (MCNS) \\
\hline Pteridaceae & Pteris exigua O.G. Martínez \& J. Prado* & DA & Martínez 881 (MCNS) \\
\hline Pteridaceae & Pteris inermis (Rosenst.) de la Sota & DA & $\begin{array}{l}\text { Martínez \& Chambi } 1919 \\
\text { (MCNS) }\end{array}$ \\
\hline Pteridaceae & Pteris multifida Poir.* & DA & $\begin{array}{l}\text { Martínez s.n. (MCNS } \\
\text { 13553) }\end{array}$ \\
\hline
\end{tabular}




\section{HELECHOS}

\begin{tabular}{|c|c|c|c|}
\hline Familia & Nombre actual & Dominio & Ejemplar de Referencia \\
\hline Pteridaceae & Pteris vittata $\mathrm{L} . *$ & $\overline{\mathrm{DA}}$ & Chambi et al. 775 (MCNS) \\
\hline Salviniaceae & Azolla cristata Kaulf. & DA-DCH & Martínez 214 (MCNS) \\
\hline Salviniaceae & Azolla filiculoides Lam. & DA-DCH & Novara 6937 (MCNS) \\
\hline Salviniaceae & Salvinia biloba Raddi & DA-DCH & $\begin{array}{l}\text { Cacharani \& Martínez } 100 \\
\text { (MCNS) }\end{array}$ \\
\hline Thelypteridaceae & $\begin{array}{l}\text { Amauropelta decurtata (Link) Salino \& } \\
\text { T.E. Almeida }\end{array}$ & DA & Martínez 871 (MCNS) \\
\hline Thelypteridaceae & $\begin{array}{l}\text { Amauropelta pachyrhachis (Kunze ex } \\
\text { Mett.) Salino \& T.E. Almeida }\end{array}$ & DA & Martínez 875 (MCNS) \\
\hline Thelypteridaceae & $\begin{array}{l}\text { Christella dentata (Forssk.) Brownsey \& } \\
\text { Jermy }\end{array}$ & DA-DCH & Martínez 354 (MCNS) \\
\hline Thelypteridaceae & Christella hispidula (Decne.) Holttum & DA-DCH & Aquino 254 (MCNS) \\
\hline Thelypteridaceae & $\begin{array}{l}\text { Thelypteris grandis A.R. Sm. var. } \\
\text { kunzeana (Hook.) A.R. Sm. }\end{array}$ & DA & Aquino 193 (MCNS) \\
\hline Thelypteridaceae & Thelypteris jujuyensis de la Sota & DA & Martínez 903 (MCNS) \\
\hline Woodsiaceae & $\begin{array}{l}\text { Woodsia montevidensis (Spreng.) } \\
\text { Hieron. var. montevidensis }\end{array}$ & DA-DCH & Martínez 1233 (MCNS) \\
\hline
\end{tabular}

Se registran 115 taxones de helechos y licófitas para el Valle de Lerma, 22 son nuevos registros respecto de la flora original, tres de los cuales se mencionan por primera vez para la flora del noroeste argentino Microgramma vaccinifolia, Pellaea atropurpurea y Pteris multifida.

En las licófitas, las familias Lycopodiaceae y Selaginellaceae tienen igual cantidad de especies. En los helechos, las familias mejor representadas, con más de diez taxones, son Pteridaceae (34), Polypodicaeae (19), Aspleniaceae (14) y Dryopteridaceae (11) (fig. 2).

La distribución de los helechos y las licófitas en los dominios fitogeográficos, indica que la mayor concentración de la diversidad se encuentra en el dominio amazónico, 73\% de los helechos y 50\% de las licófitas, y en el dominio chaqueño solo habita el $4 \%$ de los helechos, y los restantes especímenes se encuentran en ambos dominios (fig. 3). 


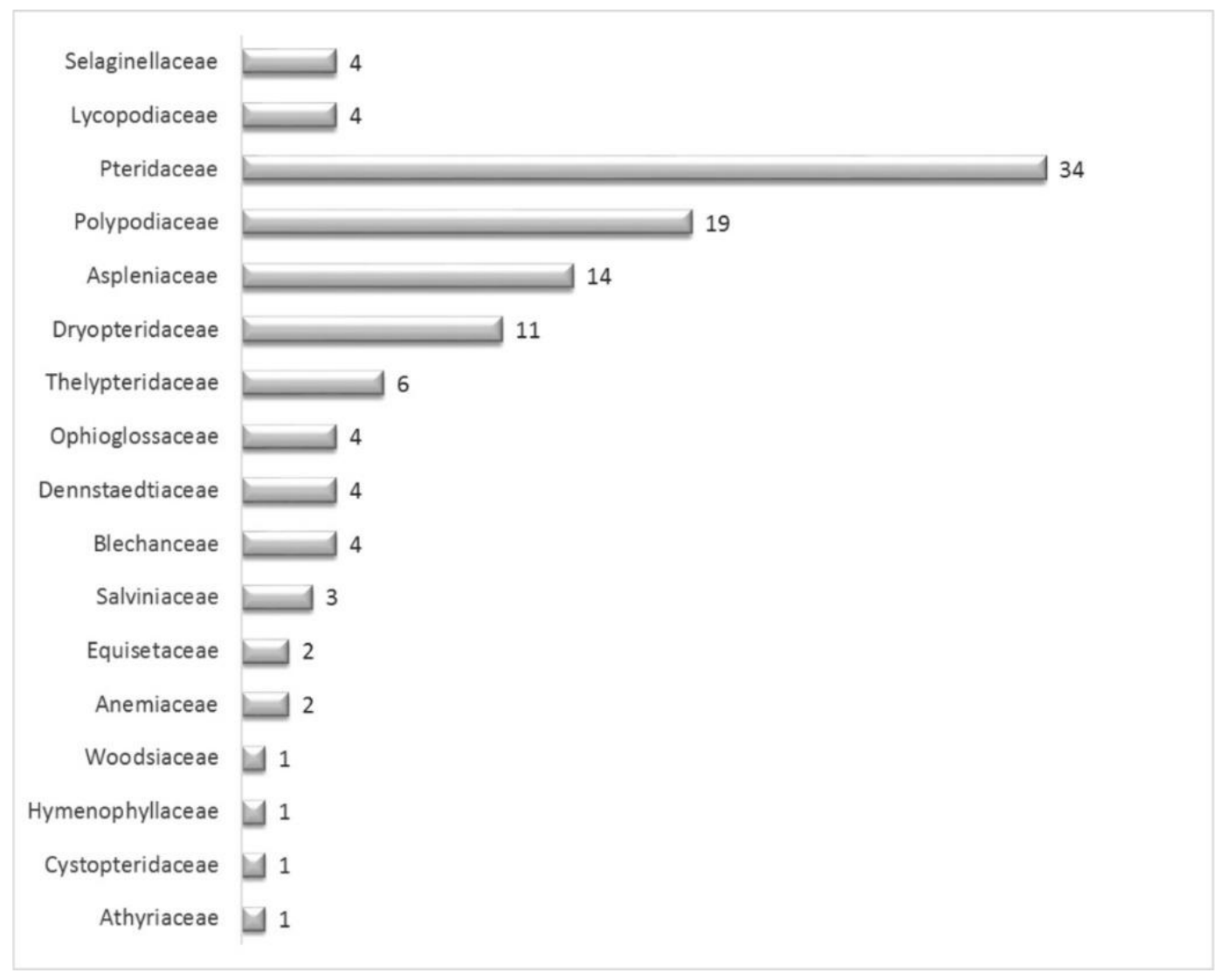

Fig. 2. Diversidad de helechos y Licófitas: datos cuantitativos de especies y taxones infraespecíficos por cada familia.

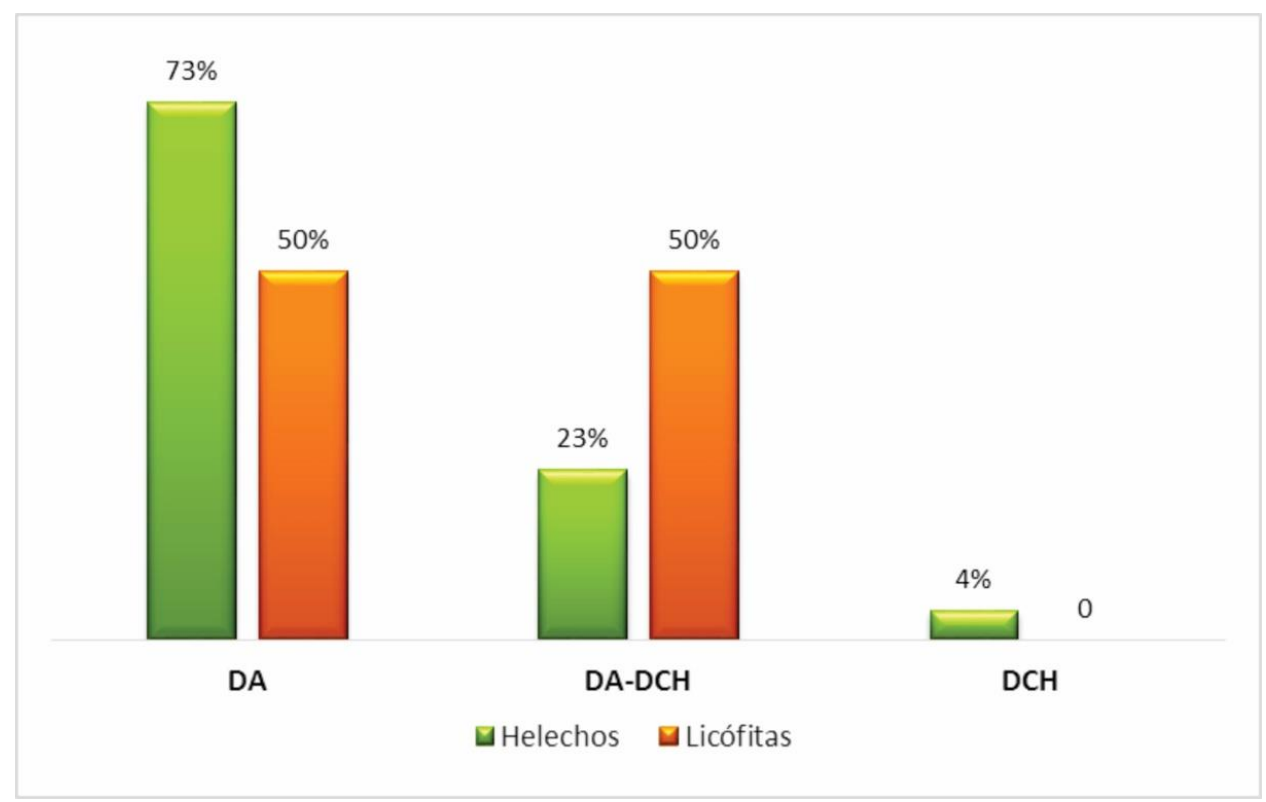

Fig. 3. Distribución de la diversidad de helechos y licófitas del Valle de Lerma en los dominios amazónico y chaqueño (DA: dominio amazónico, DA-DCH: dominio amazónico y dominio chaqueño, DCH: dominio chaqueño). 


\section{DISCUSIÓN}

La diversidad de helechos y licófitas de Flora del Valle de Lerma publicada hasta 2012, menciona 95 taxones (88 helechos y 7 licófitas), los resultados encontrados en este trabajo determinan la presencia de 115 taxones (107 helechos y 8 licófitas), de los cuales 22 corresponden a nuevos registros, ocho de éstas especies se citan por primera vez para la región estudiada (Austroblechnum penna-marina, Blechnum austrobrasilianum, B. occidentale var. occidentale, Cranfillia caudata, Microgramma vaccinifolia, Pellaea atropurpurea, Pteris multifida y Pteris vittata), tres de ellas se registran por primera vez para la región noroeste de Argentina. Los 14 taxones restantes fueron mencionados como material estudiado en el marco de otros estudios florísticos o de revisiones taxonómicas realizados por Arana \& Mynssen (2015), Condack, McHenry, Morero, Sylvestre \& Barrington (2013), Jaimez \& Martínez (2016), Jarsun, Chambi \& Martínez (2018), Kessler, Smith \& Prado (2017), Larsen, Arana, Acosta \& Ponce (2017), Meza-Torres, de la Sota \& Ferrucci (2005), Meza-Torres, Macluf, Morbelli \& Ferrucci (2015), Salino \& Semir (2002), Sanín, Martínez \& Salino (2019), Schwartsburd, Yañez \& Prado (2018) y Zuloaga \& Belgrano (2016). Según la propuesta de Gasper, Almeida, Dittrich, Smith \& Salino (2017), en el Valle de Lerma crecen cuatro de las 22 especies de la familia Blechnaceae citadas para la Argentina (Zuloaga \& Belgrano, 2016).

De los tres nuevos registros para el Valle de Lerma, la más reciente es la presencia de Microgramma vaccinifolia, especie que crece en Bolivia, Brasil, Paraguay y Uruguay, en Argentina es frecuente en las provincias del centro y nordeste (Zuloaga \& Belgrano, 2016), y su introducción se debe a su hábito epífito sobre Butia yatay (Mart.) Becc., palmera originaria del nordeste argentino que actualmente se cultiva como ornamental en paseos públicos de la ciudad de Salta, situación semejante describe Guerrero \& Delucchi (2018) para la provincia de Buenos Aires. Los otros dos helechos Pellaea atropurpurea y Pteris multifida son plantas naturalizadas, ambas se cultivan como ornamentales en Argentina.

Para la flora argentina se citan 38 taxones de licófitas y 383 de helechos (Zuloaga \& Belgrano, 2016), lo que indica que, en el Valle de Lerma, a pesar del desarrollo urbano en la región, se encuentra el 21\% (8 especies) de licófitas y el 28\% (107 taxones) de helechos de la diversidad del país.

La riqueza de helechos y licófitas encontradas en el Valle de Lerma, obedece a que en esta región se presentan dos grandes unidades de vegetación correspondientes a los dominios amazónico y chaqueño (Cabrera \& Willink, 1973). El dominio amazónico, ubicado al oeste del Valle de Lerma, donde se distinguen las Yungas australes conocidas también como Selva Tucumano-boliviana (Cabrera, 1976), constituyen el límite austral de un extenso sistema boscoso que se extiende en América del Sur desde Venezuela hasta Argentina (Brown, Grau, Malizia \& Grau, 2001). En este sistema de bosques húmedos, existente sobre las laderas montañosas, se genera el hábitat propicio de árboles porta epífitos tales como Erythrina falcata Benth. y Ocotea porphyria (Griseb.) van der Werff entre otros, que albergan la gran mayoría de las Polypodiaceae, y en el sotobosque, orilla de los cursos de agua y sobre las laderas crece la gran mayoría de los helechos, $73 \%$ de helechos y 50\% de licófitas del Valle de Lerma. En la pradera de los pastizales de altura, predomina Woodsiaceae.

El dominio chaqueño, ubicado al este del Valle de Lerma, incluye el distrito fitogeográfico del Chaco serrano (Cabrera, 1976), donde son frecuentes especies terrestres de Anemiaceae y Pteridaceae, aunque en los microambientes se encuentran Aspleniaceae, Dryopteridaceae y Selaginellaceae. En los cursos de agua de ambos dominios fitogeográficos, son frecuentes las Equisetaceae y Salviniaceae. La distribución biogeográfica de estas plantas coincide con los descrito por Martínez \& Prado (2013). 


\section{CONCLUSIONES}

Como resultado de este trabajo, se concluye que en el Valle de Lerma crecen 107 taxones de helechos en 15 familias, y 8 especies de licófitas en dos familias. Los nuevos registros respecto de la flora original incluyen 22 nuevos taxones, de los cuales tres especies se citan por primera vez para la flora de noroeste argentino, Microgramma vaccinifolia, Pellaea atropurpurea y Pteris multifida.

\section{AGRADECIMIENTOS}

Agradecemos a los curadores de los herbarios BAB, LIL, LP, MCNS y SI por facilitar la revisión de las colecciones, a los revisores anónimos por sus valiosos cometarios y al Consejo de Investigación de la Universidad Nacional de Salta (Proy. A 2344/0) por el financiamiento para este estudio.

\section{LITERATURA CITADA}

Arana, M. D. \& Mynssen, C. M. (2015). Revisión de Cystopteris (Cystopteridaceae) del Cono Sur y Brasil. Darwiniana, 3(1), 73-88. https://doi.org/10.14522/darwiniana.2015.31.639

Baudino, G. A. (1996). Hidrogeología del Valle de Lerma. Provincia de Salta, Argentina. Tesis Doctoral. Escuela del Doctorado en Ciencias Geológicas. Salta, Argentina. Universidad Nacional de Salta.

Brown, A. D., Grau, H. R., Malizia, L. R. \& Grau, A. (2001). Bosques Nublados del Neotrópico en Argentina. En A. D., Brown \& M., Kappelle (eds.). Bosques Nublados De Neotrópico (pp. 623-659). Costa Rica. INBIO.

Cabrera, A. L. (1976). Regiones fitogeográficas argentinas. En: W. F., Kugler (ed) Enciclopedia argentina de agricultura y jardinería (pp. 1-85). Buenos Aires, Argentina. Acme.

Cabrera, A. L. \& Willink, A. (1973). Biogeografía de América Latina, (1, pp. 1-192) Washington D. C. Secretaría General de la Organización de los Estados Americanos, Programa Regional de Desarrollo Científico y Tecnológico.

Condack, J. P. S., McHenry, M. A., Morero, R. E., Sylvestre, L. S. \& Barrington, D. S. (2013). Polystichum montevidense Demystified: Molecular and Morphological Data Reveal a Cohesive, Widespread South American Species. American Fern Journal, 103(2), 118130. https://doi.org/10.1640/0002-8444-103.2.118

de la Sota, E. R. \& Martínez, O. G. (1998). Polypodiaceae Bercht. et. J. Presl. En L., Novara (ed). Flora del Valle de Lerma (Salta-Argentina). Ap. Bot. Salta, ser. Flora, (5(8), pp. 1-27). Salta, Argentina. Universidad Nacional de Salta.

de la Sota, E. R \& Martínez, O. G. (2000). Hymenophyllaceae Link. En L., Novara (ed). Flora del Valle de Lerma (Salta-Argentina). Ap. Bot. Salta, ser. Flora, (6(5), pp. 1-4). Salta, Argentina. Universidad Nacional de Salta.

de la Sota, E. R., Ponce, M. M., Martínez, O. G., Giudice, G. \& Michelena, G. (2001). Pteridaceae Rchb. En L., Novara (ed). Flora del Valle de Lerma (Salta-Argentina). Ap. Bot. Salta, ser. Flora, (6(9), pp. 1-48). Salta, Argentina. Universidad Nacional de Salta.

Ganem, M. A., Giudice, E. G., Martínez, O. G. \& Sota, E. R. de la (2007). Aspleniaceae Mett. ex A. B. Frank. En L., Novara (ed). Flora del Valle de Lerma (Salta-Argentina). Ap. Bot. Salta, ser. Flora, (8(1), pp. 1-20) Salta, Argentina. Universidad Nacional de Salta.

Gasper, A. L., Almeida, T. E., Dittrich, V. A. O., Smith, A. R., \& Salino, A. (2017). Molecular phylogeny of the fern family Blechnaceae (Polypodiales) with a revised genus-level treatment. Cladistics, 33(4), 429-446. https://doi.org/10.1111/cla.12173 
Guerrero, E. L. \& Delucchi, G. (2018). Microgramma vaccinifolia en la provincia de Buenos Aires, Argentina. Historia Natural, tercera serie, 8(1), 105-108.

Jaimez, D. G. \& Martínez, O. G. (2016). Campyloneurum angustifolium, nuevo registro de Polypodiaceae para Argentina. Boletin de La Sociedad Argentina de Botánica, 51(2), 353-357. http://dx.doi.org/10.31055/1851.2372.v51.n2.14849

Jarsun, A. M., Chambi, C. J. \& Martínez, O. G. (2018). New record of Blechnum (Blechnaceae) for the flora of northwestern Argentina. Darwiniana, nueva serie, 6(2), 191-195. https://doi.org/10.14522/darwiniana.2018.62.815

Kessler, M., Smith, A. R., \& Prado, J. (2017). Prodromus of a fern flora for Bolivia. XXVII. Pteridaceae. Phytotaxa, 332(3), 201-250. https://doi.org/10.11646/phytotaxa.332.3.1

Larsen, C., Arana, M. D., Acosta, J. M. \& Ponce, M. (2017). Two new species segregated from Hymenophyllum tunbrigense (Hymenophyllaceae) in southern South America, based on morphological, anatomical, molecular and distributional evidence. Phytotaxa, 303(3), 218-232. https://doi.org/10.11646/phytotaxa.303.3.2

Martínez, O. G. (1995). Equisetaceae Richard ex Lam. \& D. C. En L., Novara (ed). Flora del Valle de Lerma (Salta-Argentina). Ap. Bot. Salta, ser. Flora, (3(13), pp. 1-9). Salta, Argentina. Universidad Nacional de Salta.

Martínez, O. G. (1996 a). Azollaceae Wettstein. En L., Novara (ed). Flora del Valle de Lerma (Salta-Argentina). Ap. Bot. Salta, ser. Flora, (4(5), pp. 1-8). Salta, Argentina. Universidad Nacional de Salta.

Martínez, O. G. (1996 b). Selaginellaceae Pal. Beauv. En L., Novara (ed). Flora del Valle de Lerma (Salta-Argentina). Ap. Bot. Salta, ser. Flora, (4(17), pp. 1-10). Salta, Argentina. Universidad Nacional de Salta.

Martínez, O. G. (1997). Lycopodiaceae P. Beauv. ex Mirbel. En L., Novara (ed). Flora del Valle de Lerma (Salta-Argentina). Ap. Bot. Salta, ser. Flora, (4(16), pp. 1-8). Salta, Argentina. Universidad Nacional de Salta.

Martínez, O. G. (1998). Ophioglossaceae (R. Br.) C. Agardh. En L., Novara (ed). Flora del Valle de Lerma (Salta-Argentina). Ap. Bot. Salta, ser. Flora, (5(3), pp. 1-8). Salta, Argentina. Universidad Nacional de Salta.

Martínez, O. G. \& Cacharani, D A. (2011). Salviniaceae Dumortier En L., Novara (ed). Flora del Valle de Lerma (Salta-Argentina). Ap. Bot. Salta, ser. Flora, (11(1), pp. 1-5). Salta, Argentina. Universidad Nacional de Salta.

Martínez, O. G. \& de la Sota, E. R. (2000 a). Dennstaedtiaceae Link. En L., Novara (ed). Flora del Valle de Lerma (Salta-Argentina). Ap. Bot. Salta, ser. Flora, (6(7), pp. 1-11). Salta, Argentina. Universidad Nacional de Salta.

Martínez, O. G. \& de la Sota, E. R. (2000 b). Hymenophyllaceae Link. En L., Novara (ed). Flora del Valle de Lerma (Salta-Argentina). Ap. Bot. Salta, ser. Flora, (6(5), pp. 1-4). Salta, Argentina. Universidad Nacional de Salta.

Martínez, O. G. \& de la Sota, E. R. (2005). Lomariopsidaceae Alston. En L., Novara (ed). Flora del Valle de Lerma (Salta-Argentina). Ap. Bot. Salta, ser. Flora, (7(8), pp. 1-12). Salta, Argentina. Universidad Nacional de Salta.

Martínez, O. G. \& Prado, D. E. (2013). Distribución Fitogeográfica de Helechos y Licófitas en el Valle de Lerma (Salta-Argentina). Chloris Chilensis, 16(2), 1-19. Retrieved from http://www.chlorischile.cl/helechos del valle Lerma-prado/Helechos valle Lerma.htm

Martínez, O. G., de la Sota, E. R. \& Aquino, V. H. (2006). Vittariaceae (C. Presl) Ching. En L., Novara (ed). Flora del Valle de Lerma (Salta-Argentina). Ap. Bot. Salta, ser. Flora, (7(17), pp. 1-4). Salta, Argentina. Universidad Nacional de Salta.

Martínez, O. G., de la Sota, E. R. \& Narváez, P. L. (2003). Schizaceae Kaulf. En L., Novara (ed). Flora del Valle de Lerma (Salta-Argentina). Ap. Bot. Salta, ser. Flora, (7(2), pp. 1-6). Salta, Argentina. Universidad Nacional de Salta.

Martínez, O. G., Sota, E. R. de la \& Narváez, P. L. (2004). Grammitidaceae (C. Presl) Ching. En L., Novara (ed). Flora del Valle de Lerma (Salta-Argentina). Ap. Bot. Salta, ser. Flora, (7(5), pp. 1-5). Salta, Argentina. Universidad Nacional de Salta. Universidad Nacional de Salta. 
Recibido:

23/marzo/2019

Aceptado:

06/diciembre/2019
Meza-Torres, E. I., de la Sota, E. R. \& Ferrucci, M. S. (2005). Adiciones a la Flora Pteridofítica del NE Argentino. Boletín de la Sociedad Argentina de Botánica, 40 (Supl.), 194-195. Córdoba, Argentina. Sociedad Argentina de Botánica.

Meza-Torres, E. I., Macluf, C. C., Morbelli, M. A., \& Ferrucci, M. S. (2015). The circumscription of problematic species of Ophioglossum (Ophioglossaceae) from Southern South America: A palynological approach. Phytotaxa, 205(3), 145-156. https://doi.org/10.11646/phytotaxa.205.3.2

Mynssen, C. M. (2011). Woodsiaceae (Hook.) Herter (Polypodiopsida) no Estado do Rio Grande do Sul, Brasil. Pesquisas Botânica, 62(1), 273-297. Retrieved from http://www.anchietano.unisinos.br/publicacoes/botanica/botanica62/11.pdf

Olson, D. M., Dinerstein, E., Wikramanayake, E. D., Burgess, N. D., Powell, G. V. N., Underwood, E. C., D’Amico, J. A., Itoua, I., Strand, H., Morrison, J. C., Loucks, C. J., Allnutt, T. F., Ricketts, T. H., Kura, Y., Lamoreux. J. F., Wettengel, W. W., Hedao, P. \& Kassem, K. R. (2001). Terrestrial Ecoregions of the World: A New Map of Life on Earth. BioScience, 51(11), 933-938. https://doi.org/10.1641/0006-3568

Prado, D. E. \& Gibbs, P. E. (1993). Patterns of Species Distribution in the Dry Seasonal Forest of South America. Annals of the Missouri Botanical Garden, 80(4), 902-927. Retrieved from http://www.biodiversitylibrary.org/item/89029

Ponce, M. M. \& Martínez, O. G. (2008). Thelypteridaceae Ching ex Pic. Serm. En L., Novara (ed). Flora del Valle de Lerma (Salta-Argentina). Ap. Bot. Salta, ser. Flora, (8(14), pp. 1-19). Salta, Argentina. Universidad Nacional de Salta.

Ponce, M. M. \& Martínez, O. G. (2012). Dryopteridaceae Herter, nom. cons. En L., Novara (ed). Flora del Valle de Lerma (Salta-Argentina). Ap. Bot. Salta, ser. Flora, (11(8), pp. 1-23). Salta, Argentina. Universidad Nacional de Salta.

Salino, A. \& Semir, J. (2002). Thelypteridaceae (Polypodiophyta) do Estado de São Paulo: Macrothelypteris e Thelypteris subgêneros Cyclosorus e Steiropteris. Lundiana, 3(1), 9-27. Retrieved from http://cncflora.jbrj.gov.br/plataforma2/arquivos/biblio/50b65b8 8534f8_50b3aff5b76d5_4f609a9978d2e_SalinoSemir2002ThelypteridaceaeSPMacrot helypteris.pdf

Sanín, D., Martínez, O. G. \& Salino, A. (2019). New record of Serpocaulon triseriale (Sw.) A.R. Sm. (Polypodiaceae) in Argentina, with morphological comparison of relatives. Check List, 15(1), 175-180. https://doi.org/10.15560/15.1.175

Schwartsburd, P. B., Yañez, A. \& Prado, J. (2018). Formal recognition of six subordinate taxa within the south american bracken fern, Pteridium esculentum (P. esculentum subsp. arachnoideum s.l.-Dennstaedtiaceae), based on morphology and geography. Phytotaxa, 333(1), 22-40. https://doi.org/10.11646/phytotaxa.333.1.2

Thiers, B. (2019). Index Herbariorum: A global directory of public herbaria and associated staff. New York Botanical Garden's Virtual Herbarium. tp://sweetgum.nybg.org/science/ih/ (consultado en marzo de 2019).

Zuloaga, F. O. \& Belgrano, M. (Eds). (2016). Flora Vascular de La República Argentina. Licófitas, Helechos y Gymnospermae (2, pp. 235-271). Buenos Aires, Argentina. Estudio Sigma S.R.L. 